\title{
Análisis antropométrico de la mezquita-catedral de Córdoba
}

\section{Anthropometric Analysis of the Mosque-Cathedral of Cordoba}

\author{
F. J. Roldán-Medina ${ }^{(*)}$
}

\section{RESUMEN}

Este estudio forma parte de una investigación más amplia sobre metrología histórica de la arquitectura. Se pretende en primer lugar divulgar los principios científicos del primer método técnico desarrollado para analizar las modulaciones de obras realizadas con el antiguo sistema antropométrico. Como aplicación práctica se presentan parte de los resultados obtenidos en la mezquita-catedral de Córdoba, un complejo ejemplo entre los numerosos edificios analizados hasta ahora que responden al mismo sistema milenario de control métrico. Para el análisis se utiliza el método de dimensionado por doble escala $\sqrt{ }_{2}$ sobre un levantamiento del alzado oeste del edificio realizado por Antonio Almagro Gorbea, complementado con otras bases gráficas y mediciones directas. Se destaca la utilización de un único codo hispano-musulmán frente a 3 varas distintas en los añadidos cristianos, las diferencias entre sillerías emirales y califales, la composición estática de portadas, y la modulación dinámica de otros numerosos elementos.

Palabras clave: Modulación; proporción; metrología; historia de la arquitectura; construcción; mezquita-catedral de Córdoba.

\section{ABSTRACT}

This study forms part of a more extensive research on metrology in historic architecture. Its primary intention is to disseminate the scientific principles of the first technical method that was developed to analyze the modulations of works that were carried out with the old anthropometric system. As a practical application, parts of the results obtained in the CathedralMosque of Cordoba are also presented, a complex example among many others analyzed, corresponding to buildings of millenarian metric system. To perform the analysis I used the Method of Modulation and Sizing of Historic Architecture applied to a survey of the west elevation of the building executed by Antonio Almagro, supplemented with other graphic bases and direct measurements. The use of a unique Hispanic Muslim cubit stands out next to 3 distinct rods in the Christian additions. Differences can also be appreciated among the emiral and califal ashlar masonry, as well as the static composition in the entrances. Also highlighted are the dynamic modulations of numerous other elements in the building.

Keywords: Modulation; proportion; metrology; architectural history; construction; Mosque-Cathedral of Cordoba.

(*) Universidad de Granada (España)

Persona de contacto/Corresponding author: froldan@ugr.es (F. J. Roldán-Medina).

Cómo citar este artículo/Citation: Roldán-Medina, F. J. (2015). Análisis antropométrico de la mezquita-catedral de Córdoba. Informes de la Construcción, 67(539): e093, doi: http://dx.doi.org/10.3989/ic.14.024.

Licencia / License: Salvo indicación contraria, todos los contenidos de la edición electrónica de Informes de la Construcción se distribuyen bajo una licencia de uso y distribución Creative Commons Reconocimiento no Comercial 3.o. España (cc-by-nc). 


\section{INTRODUCCIÓN}

Pitágoras, Plotino o San Agustín opinaban que la belleza es tanto más elevada cuanto más sencilla sean las relaciones matemáticas (1). Sobre la necesidad de que todo se reduzca necesariamente a número, peso y medida nos habla Pacioli en la Divina Proporción (2). Del control dimensional de las obras de construcción se han ocupado autores como Neufert (3), Aguirre de Yraola (4), Kurent (5), o Schmitt y Heene (6).

Sobre sistemas de medidas del pasado en general, y concretamente sobre los orígenes y vicisitudes del antiguo y universal sistema antropométrico, existen interesantes trabajos de Kula (7), Michell (8), Escalona (9), o Suárez Jiménez (10) entre otros.

Sobre las proporciones y medidas de las antiguas construcciones existe una bibliografía muy variada, tanto histórica como contemporánea. Comenzando por el mítico texto de Vitruvio (11), los numerosos tratados surgidos a partir del Renacimiento (12), y un conjunto de familias de teorías de la proporción en arquitectura que crece continuamente desde hace siglos ${ }^{1}$.

Concretamente sobre la mezquita de Córdoba existen numerosas referencias bibliográficas en general ${ }^{2}$, así como una serie de estudios metrológicos y de proporciones que han tenido una gran repercusión en posteriores trabajos ${ }^{3}$.

Sobre métodos científicos para analizar modularmente un edificio del pasado no se conocen técnicas aceptadas que lo permitan, más allá de la detección de cuadrículas. Y dado que en la actualidad no podemos expresar en números las proporciones de la mayoría de las obras del pasado, nuestro saber es deficiente e insatisfactorio en este campo ${ }^{4}$.

Pasa subsanar esta carencia se ha propuesto a la comunidad científica un método práctico para analizar correctamente obras realizadas con el antiguo sistema de medidas.

Concretamente el método permite:

- Detectar el sistema de proporciones de una obra

- Establecer la modulación antropométrica de cada parte

- Reconstruir las tramas modulares generales y establecer con precisión el módulo específico de la obra.

- Determinar el tamaño y posición teórica de cada elemento

- Agrupar modulaciones parciales y detectar grandes módulos

- Comprobar el mantenimiento de métrica en el análisis de elementos secundarios

- Detectar métricas distintas a la general (añadidos y reformas)

- Analizar deformaciones e irregularidades

- Clasificar ámbitos (zonas estáticas y dinámicas, espesores de muro, radios y peraltes de arco...)

- Analizar aproximaciones a proporciones notables del conjunto y sus partes

- Construir patrones con la métrica de la obra, comprobando y ampliando los resultados in situ
- Confrontar métricas de obras coetáneas

- Generar modelos tridimensionales con la geometría ideal de la obra, o sus restos.

- Añadir a estos modelos virtuales hipotéticas partes desaparecidas según la métrica de la obra

Excepto de los dos últimos puntos, se presentan aquí resultados obtenidos en el estudio de la mezquita-catedral de Córdoba, un conjunto monumental que constituye tanto la mezquita fundacional como 3 grandes ampliaciones islámicas, más todos los añadidos cristianos posteriores incluido el crucero de la catedral, y cuyas obras se desarrollaron durante casi un milenio.

\section{LAS BASES DE REFERENCIA MÉTRICA}

Se inició el estudio utilizando el levantamiento fotogramétrico publicado por Antonio Almagro del exterior de la Puerta de los Visires o de San Esteban (31). Posteriormente el mismo Antonio Almagro ha facilitado el levantamiento fotogramétrico de la totalidad del alzado oeste de la catedral ex-mezquita, trabajo realizado en 2011 y aún inédito. Este rigoroso documento gráfico ha constituido la base métrica para establecer la modulación de la totalidad de este frente del edificio, el más significativo históricamente.

Para interpretar la modulación de las dimensiones generales se ha utilizado la planta publicada también por Antonio Almagro (32). En el análisis del alzado interior de la puerta de los Deanes se ha utilizado el levantamiento realizado por Pedro Marfil en 2010. Las secciones y alzados publicados por Félix Hernández en 1975 han servido de referencia en el estudio de los alminares de Hisam I y de Abd al-Rahmán III.

\section{EL MÉTODO DE DIMENSIONADO}

El procedimiento de análisis métrico adoptado se dedujo del estudio geométrico sobre un levantamiento fotogramétrico de un palacio nazarí (33), y ha sido corroborado por el autor tanto aquí como en otras numerosas obras. Se basa en un sistema de medidas con un único módulo antropométrico por cada obra, y con un doble sistema de unidades duodecimales en proporción raíz cuadrada de dos $\left(V_{2}\right)$, la relación que existe entre la diagonal y el lado de un cuadrado. Estas 2 escalas armónicas se utilizan independientemente para generar cuadrículas en diseño estático, o bien se usan combinadamente produciendo tramas dinámicas bi-escalares (Figura 1). Por lo tanto este método modular permite componer correctamente los numerosos diseños Ad Quadratum y composiciones octogonales tan documentados en la arquitectura histórica, así como generar otras tramas ortogonales formadas por cuadrados, rectángulos $\sqrt{2}_{2}$ y rectángulos de plata (34).

Se aprovecha la capacidad de llevar a cabo el control métrico de las obras mediante simples escuadras en proporción antropométrica para representar y cuantificar las distintas dimensiones sobre la base métrica (35). Las frecuentes modulaciones dinámicas requieren de pares de valores enteros (binomios) para su correcta expresión numérica.

\footnotetext{
${ }^{1}$ Entre los textos que analizan o recopilan parte de la extensa historiografía sobre la teoría de la proporción en arquitectura destacamos: (13) (14) (15) (16) y (17).

2 Se puede consultar: (18) (19) (20) y (21).

3 Nos referimos a trabajos como: (22) (23) (24) (25) (26) (27) (28) y (29).

4 Esto opinaba Lord Kelvin de lo que no se podía expresar con números, en una cita tomada de (30).
} 


\title{
SISTEMA DE MEDIDAS CON DOBLE ESCALA $\sqrt{2}$
}

\section{UNIDAD BÁSICA}

\author{
homo
}

\section{SISTEMA DE UNIDADES}



\section{MODULACIONES LINEALES}

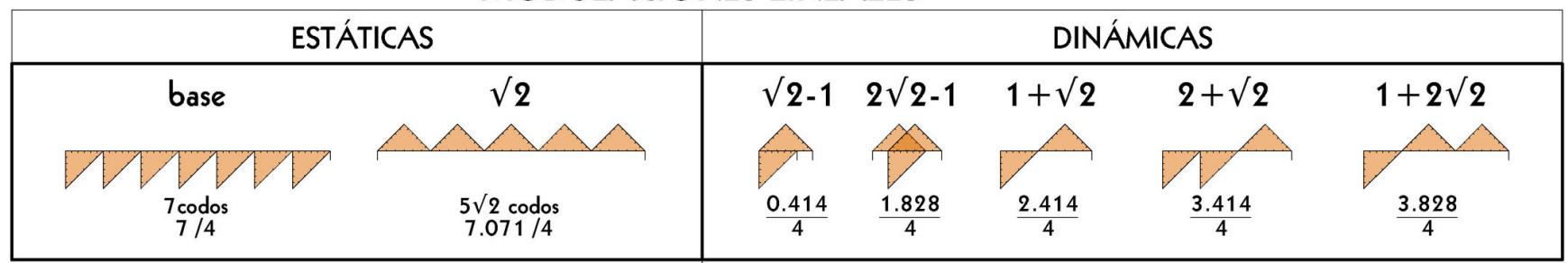

\section{TRAMAS MODULARES}

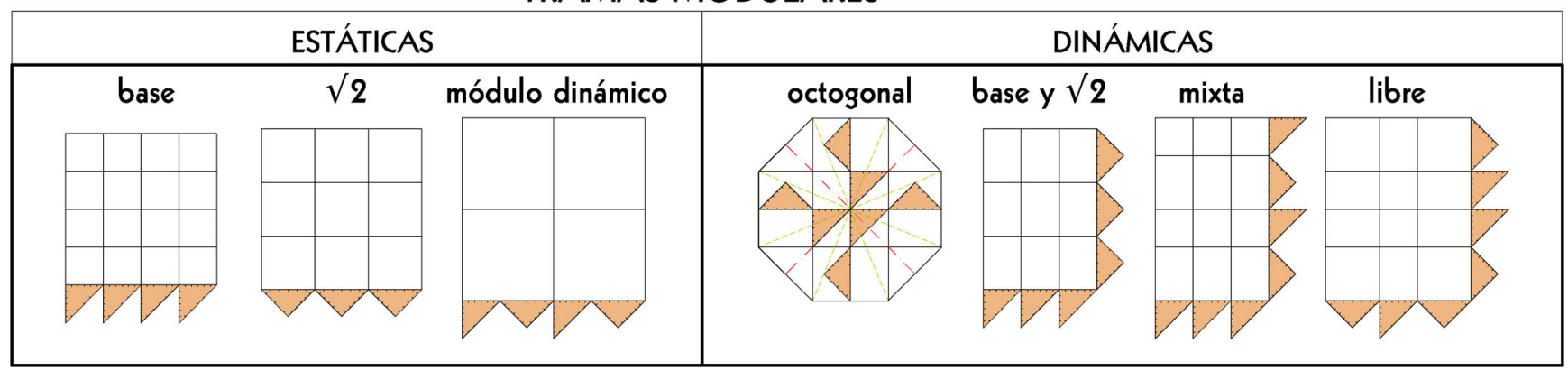

Figura 1. Sistema de medidas con doble escala.

En base a estos principios se deben deducir las proporciones entre las distintas partes de una obra hasta completar la trama modular de sus dimensiones generales. Al ajustar esta trama teórica a la mayor medida real se obtiene el módulo o unidad básica de la construcción. Con este valor y el doble sistema de unidades se deben justificar también los tamaños de los elementos secundarios de la misma obra, por análisis gráfico de la base métrica y/o mediante mediciones directas de la obra original con patrones de doble graduación construidos a escala.

\section{LAS DIMENSIONES GENERALES}

Se han detectado 4 módulos distintos (Figura 2) utilizados en la construcción del alzado oeste de la mezquita-catedral de Córdoba:
Módulo 1: Corresponde con el oratorio de la mezquita-aljama. Su valor antropométrico es de una braza de 158,37 cm, lo que le corresponde un codo de $39,59 \mathrm{~cm}$.

Módulo 2: Se ha detectado este patrón en el trazado exterior de la Puerta de los Deanes. El valor de su vara es 104,82 cm (209,69 cm de braza).

Módulo 3: Desde la Puerta de los Deanes hasta la esquina norte, incluido el Postigo de la Leche, y en otras zonas puntuales del oratorio, se utilizó un patrón de valor antropométrico $165,23 \mathrm{~cm}$, es decir, una vara de $82,62 \mathrm{~cm}$ o un codo de $41,31 \mathrm{~cm}$.

Módulo 4: La zona correspondiente a la Capilla de la Inmaculada Concepción, en el centro del alzado, fue ejecutada utilizando un módulo de $170,48 \mathrm{~cm}$, lo que le corresponde una vara de $85,24 \mathrm{~cm}$. 


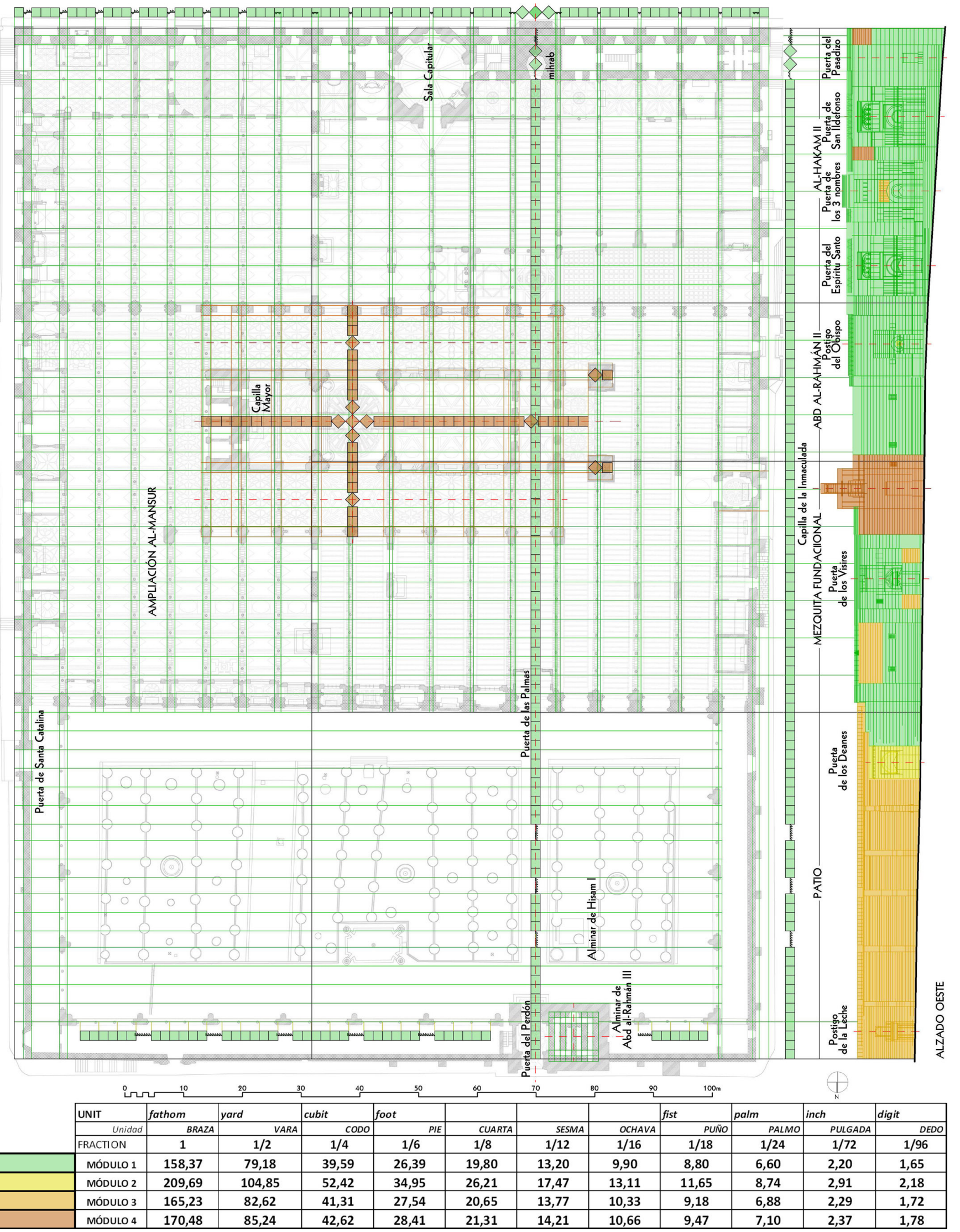

Figura 2. Modulación de las dimensiones generales. 


\subsection{Mezquita}

La zona sur del alzado oeste, la que corresponde con la ampliación de al-Hakam II, es la que presenta mayor adaptación a la trama estática en base al Módulo 1. A partir del doble muro de la qibla se detecta una modulación de valor 8 brazas entre los ejes de las 3 puertas, y centrados entre ellas avanzan los contrafuertes. Este ritmo de 4 brazas se conserva, algo descentrado en el Postigo del Obispo, hasta el eje de la espadaña cristiana de la Capilla de la Inmaculada.

Cuando se ha confrontado esta cadencia con la planta general del edificio se ha detectado una concordancia de 2 brazas $(316,74 \mathrm{~cm})$ entre ejes de soportes de las arcadas de las naves. E igual espesor tiene la fachada al patio, con lo que se cuentan 34 intervalos exactos de 2 brazas hasta el mihrab.

El doble muro de la qibla no se ajusta a esta trama estática impuesta en las arcadas de las naves, sino que se recurre a una modulación dinámica de $1+2 \sqrt{ }_{2}$ codos para determinar el espesor de los muros. Y entre ellos se dimensiona con un ancho de $2 \sqrt{ }_{2}$ brazas el ámbito donde se localiza el mihrab, la cámara del tesoro y la puerta del pasadizo.

En dirección sur el trazado del Patio de los Naranjos no responde a Módulos 1 enteros, pero si se adapta a fracciones binarias con un total de 149 codos. En total suma la longitud teórica de alzado oeste $423+12 \sqrt{ }_{2} \operatorname{codos}$, lo que equivale a unos 440 codos y a $174,18 \mathrm{~m}$.

En sentido perpendicular y considerando un espesor de arcada de valor $\sqrt{ }_{2}$ codo, obtenemos para las 8 naves intermedias, simétricas respecto al mihrab, un ancho neto de 4 brazas. La nave central del mihrab se ajusta a $3 \sqrt{ }_{2}$ brazas de frente, más $\sqrt{ }_{2}$ codo hasta los soportes. Y en las extremas las 4 brazas de ámbito se miden desde la línea de fachada, por lo que hay que deducir el espesor de los muros fijado en $2 \sqrt{ }_{2}$ codos. Ello arroja un ancho libre para las naves extremas de $16-2 \sqrt{ }_{2}$ co$\operatorname{dos}(5,21 \mathrm{~m})$.

Esto es válido para las naves de la mezquita fundacional, y para sus prolongaciones en las ampliaciones de Abd al-Rahmán II y al-Hakam II. En cuanto a la última ampliación de alMansur se acorta el ancho neto de las naves a 3 brazas mientras se amplía el espesor de las arcadas a $2+\sqrt{ }_{2}$ codos. Esto se cumple en todas estas naves excepto en la primera adosada al muro de la primitiva fachada, desde la que se miden 4 brazas enteras incluida la primera arcada hasta la segunda nave.

El ancho total de la mezquita fundacional -incluidos sus muros pero no los contrafuertes- es por tanto de $160+23 \sqrt{2}_{2}$ codos $(76,22 \mathrm{~m})$. La ampliación de al-Mansur añade $114+7 \sqrt{ }_{2}$ codos $(49,05 \mathrm{~m})$. El ancho total de la mezquita-catedral en sentido este-oeste es $274+30 \sqrt{ }_{2}(125,28 \mathrm{~m})$, valor muy próximo a 315 codos.

\subsection{Catedral}

Se han ensayado los módulos detectados en la fachada oeste sobre la planta del crucero de la catedral construida en el interior de la mezquita, encontrándose correspondencia con el fijado para la Capilla de la Inmaculada Concepción (Modulo 4). Utilizando su valor podemos justificar las dimensiones del crucero de la catedral en sentido norte-sur con $4+3 \sqrt{ }{ }_{2}$ brazas, y para las naves laterales $4+\sqrt{ }_{2}$ brazas cada una. En sentido este-oeste el crucero se ajusta a $2+3 \sqrt{ }_{2}$, prolongándose la nave central hacia el este 7 brazas enteras del altar mayor, y 2 brazas de las capillas traseras. Hacia el coro 3 naves intermedias de la mezquita se salvan con 4 brazas por nave. La nave central más ancha de la mezquita se salvaría con $2,5+\sqrt{ }_{2}$ brazas en el tras-coro, y hasta las torres de planta cuadrada (de lado $1+\sqrt{ }_{2}$ brazas) otra nave se salva con 2,5 brazas. Contando con 1 braza por cada línea de soportes obtenemos un total de $16+5 \sqrt{ }_{2}$ brazas para el ancho total $(39,33 \mathrm{~m})$, y de $34+5 \sqrt{ }_{2}$ brazas para el largo (70,02 m).

\section{LOS ELEMENTOS DEL ALZADO OESTE (Figura 3)}

\subsection{Sillerías}

En la zona central de la fachada oeste, a partir de la Puerta de los Deanes y hasta la Puerta del Espíritu Santo -exceptuando la Capilla de la Inmaculada Concepción y puntualmente otras superficies- los sillares de los lienzos de muro y de los 5 contrafuertes existentes se disponen generalmente en hiladas de altura $\sqrt{ }_{2}$ codo del Módulo 1 (unos $56 \mathrm{~cm}$ ), si bien también hay otras dimensionadas en palmos, en $\sqrt{ }_{2}$ palmos, o en combinación de ambas escalas.

En contraste en la zona sur correspondiente a la ampliación de al-Hakam II predomina una altura de sillar de valor $2+V_{2}$ cuartas $(67,6 \mathrm{~cm})$ del mismo módulo, e igualmente aparecen otros valores por combinación dinámica.

A partir de la Puerta de los Deanes hasta el extremo norte el cerramiento del patio dispone de una altura de sillares establecida en palmos enteros del Módulo 3, generalmente 5 palmos o 1 codo. La misma métrica se detecta en la fábrica que se extiende por encima de la Puerta de los Deanes, en toda la crestería gótica que corona esta parte mediante módulos de 9 palmos de ancho, en la parte superior del lienzo con dos ventanas al norte de la Puerta de los Visires, y en las zonas bajas a ambos lados de dicha puerta.

La fachada de la Capilla de la Inmaculada Concepción está realizada con fábrica de 1 codo de altura correspondiente al Módulo 4. La misma métrica, aunque con dimensiones más pequeñas en palmos o medios palmos, se detecta en la parte superior del contrafuerte entre la Puerta de los 3 nombres y la Puerta de San Ildefonso, y en la misma zona del último contrafuerte en el extremo sur del alzado.

\subsection{Puertas de al-Hakam II}

Todos los anchos de los diferentes elementos de estas 3 puertas pueden establecerse en palmos enteros del Módulo 1. Las alturas a partir del hueco central también se determinan en general de igual manera, pero hasta el centro del arco se modula dinámicamente al igual que la sillería de los muros.

La dimensión de huecos centrales y dinteles adovelados coincide en los 3 casos. El ancho de los huecos es 26 palmos, su altura $24+15 \sqrt{ }_{2}$, y la de los dinteles $6+9 \sqrt{2}_{2}$.

También coincide la distancia entre contrafuertes de las portadas laterales (26 codos), mientras que la central es algo mayor y alcanza los 40 pies.

El resto de elementos en las 3 portadas presenta similar composición. Sin embargo sus dimensiones varían de una a otra. 

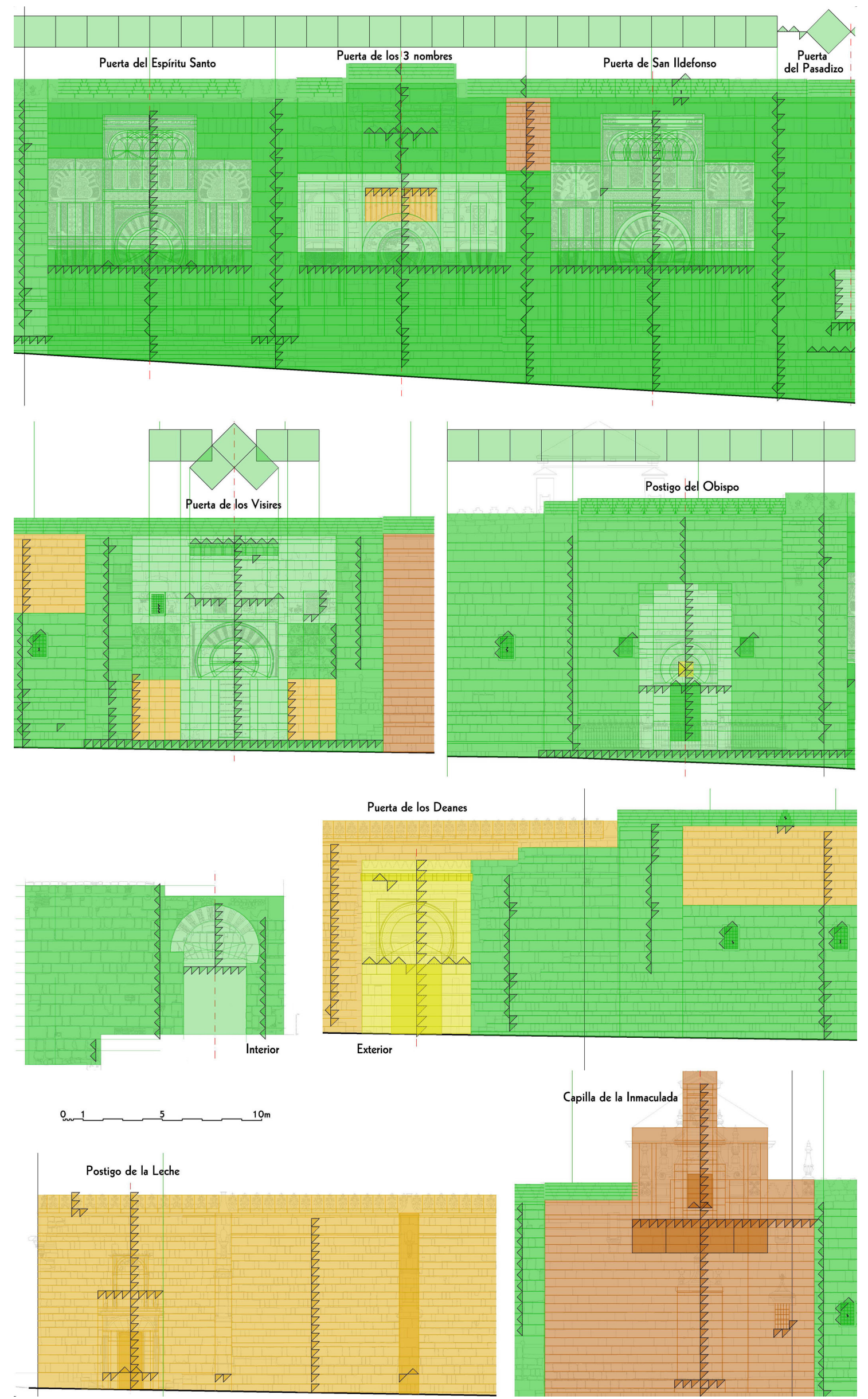

Figura 3. Modulación del alzado oeste. 
Así las jambas en la Puerta de San Ildefonso miden 4 palmos de ancho, y en los otros casos 5 . El cuerpo central tiene un ancho de 80 palmos en la Puerta de San Ildefonso, 14 codos en la Puerta de los 3 nombres, y 3 brazas en la Puerta del Espíritu Santo.

En las portadas laterales el alfiz se remata a 2 brazas sobre el centro del intradós del arco. Este elemento y el resto de decoración no se conservan en la portada central. No obstante en su parte superior sobresale una zona almenada que coincide con el ancho del cuerpo central, más alto que en las portadas laterales. Debajo aparece la impronta de unos pequeños huecos de $6 V_{2}$ de ancho, separados entre sí 2 palmos. Además se detecta que las columnas de decoración gótica existente sobre el arco están separadas 1 pie del Módulo 3.

\subsection{Postigo del Obispo}

Varía sustancialmente el proceso de modulación, pero manteniendo el Módulo 1. El ancho del hueco se establece en $1+2 V_{2}$ codos, y el de sus jambas en 13 palmos. No dispone de cuerpos laterales. La distancia entre contrafuertes vuelve a ser 26 codos.

En el centro del arco se localiza una lápida cuadrada del obispo Don Juan Daza, cuyo lado coincide con $\sqrt{ }_{2}$ codos del Módulo 2.

Actualmente la puerta se remata a 10 palmos sobre la clave exterior del arco dovelado, pero se aprecia encima una zona que presenta distinto despiece de sillería que el resto del lienzo. Se trata de 7 hiladas de 5 palmos de altura cada una, que se prolongan 1 braza a cada lado de las jambas y se escalonan superiormente.

\subsection{Puerta de los Visires}

Casi todas las dimensiones horizontales y verticales se pueden establecer en palmos enteros del Módulo 1. El ancho del hueco de acceso es 24 ( 1 braza), las jambas tienen 6 (1 codo), y 23 más existen al extremo de la portada. Hasta la vertical de las dos ventanas laterales existen 12, y el ancho de éstas también es 12. Restan otros 13 palmos hasta los contrafuertes, con lo que el ancho total del lienzo es también 26 codos.

No obstante un curioso juego dinámico también puede justificar las dimensiones generales en brazas enteras. Así la braza del ancho del hueco central se centraría en un ancho total del alfiz de $2 \sqrt{ }_{2}$, lo mismo que la cornisa. El ancho de la portada correspondería a $2+\sqrt{ }_{2}$, y la disposición de las ventanas laterales añadiría otra braza a cada lado.

La altura del hueco de entrada es 44 palmos, y su dintel tiene 6. Sobre él se localiza a 8 el centro del trasdós. Hasta la clave del arco del trasdós hay 12 (diámetro 60), 2 tiene la franja del recuadro, y sobre él se localizan las ventanas a 4. Las improntas de los tres huecos situados sobre el arco dejan entre sí unos paneles decorados de 12 de altura, y sobre ellos a 33 se dispone una cornisa de 9 de altura. La disposición horizontal de sus ménsulas parece responder a un ritmo $\sqrt{ }_{2}$, mientras que su anchura es de 3 .

Mientras los sillares labrados existentes a ambos lados del arco tienen $\sqrt{ }_{2}$ codos de altura, como los contrafuertes y res- tos de paños de esta zona, la parte superior se dimensiona en palmos enteros.

Volvemos a mencionar que la sillería a ambos lados de las jambas de la puerta está dimensionada mediante palmos enteros del Módulo 3.

\subsection{Puerta de los Deanes}

Dimensionada exteriormente mediante el Módulo 2. El ancho de su hueco responde a $2+\sqrt{ }_{2}$ codos, el remarco del arco se prolonga $\sqrt{ }_{2}$ y otro tanto el extremo de la portada, que en total dispone de un ancho de $2+6 \sqrt{ }_{2}$ codos.

Las jambas disponen de sillares de 1 codo de altura. El dintel adovelado es de 2 codos, y sobre él se dispone a 2 palmos el único centro de sus arcos. A 15 palmos se localiza la clave del intradós (diámetro 30), y 7 palmos de espesor tiene el arco hasta la clave de su trasdós (diámetro 44). Sobre él a 2 codos se dispone una cornisa de 1 codo de altura, y se remata la portada con unas pequeñas almenas de otro codo de altura. Las ménsulas de la cornisa tienen un ancho de $1+V_{2}$ palmos, y están separadas $4^{1 / 2}$ palmos.

Analizando el alzado interior se detecta la continuidad del Módulo 1, tanto en las sillerías de ambos lados como en el trazado general de la puerta. El ancho del hueco corresponde con 2 brazas, el dintel adovelado con 2 codos, el radio del intradós con 1 braza. El trasdós se peralta 2 palmos y dispone de un radio de 34 palmos.

\subsection{Postigo de la Leche}

Portada dimensionada con el Módulo 3. El ancho del hueco de acceso es $2 V_{2}$ codos, las jambas hasta las semicolumnas tienen 8 palmos, y éstas tienen 4 hasta completar el ancho de la portada $\left(4+2 \sqrt{2}_{2}\right)$.

El hueco de la puerta dispone de 7 codos de altura, el dintel tiene 2, y otro la cornisa de transición hasta el cuerpo superior. Éste está compuesto por un hueco ciego enmarcado, con un ancho total de 26 y una altura de 38 palmos.

\subsection{Capilla de la Inmaculada Concepción}

La obra de esta capilla -que ofrece fachada en el alzado oeste de la mezquita catedral- está dimensionada mediante el Módulo 4. Todos sus elementos se ajustan a modulación estática, a excepción de la ventana superior que parece presentar un ancho de hueco de $2 \sqrt{ }_{2}$ codos. La sillería es regular de 1 codo de altura. El nicho sobresaliente del altar presenta 8 pies de ancho. La ventana del contrafuerte donde se ubica la sacristía dispone de 2 codos de ancho por 3 de alto, protegida por una reja de barrotes separados 2 palmos de ancho por 3 $1 / 2$ alto. El ancho exterior del lucero de la capilla se ajusta a 4 brazas.

\section{ALMINARES (Figura 4)}

\subsection{Alminar de Hisam I}

Los alzados publicadas en 1975 por Félix Hernández sobre los restos del primitivo alminar revelan que fue construido según una planta cuadrada de $1+2 \sqrt{2}_{2}$ brazas del Módulo 1 $(6,06 \mathrm{~m})$. 

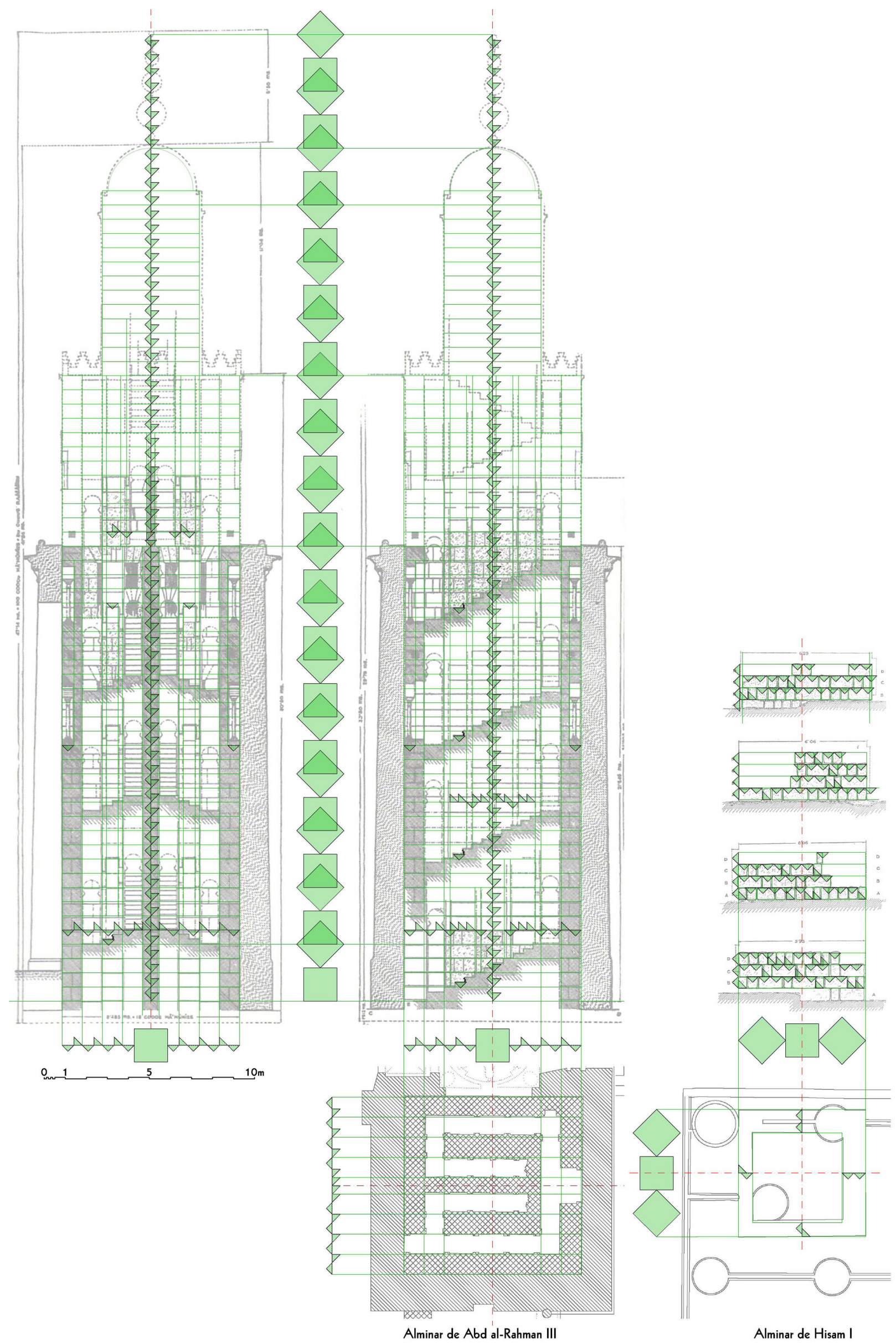

Figura 4. Modulación de los alminares. 
Las hiladas de sillería tienen un alto constante de $\sqrt{2}_{2}$ codos. La longitud de los sillares a soga se dimensiona en $\sqrt{ }_{2}$ palmos, predominando los de $2 \sqrt{ }_{2}$ codos. Los colocados a tizón presentan tanto palmos como $\sqrt{ }_{2}$ palmos, siendo la mayoría de 1 codo o de $5 \sqrt{ }_{2}$ palmos.

\subsection{Alminar de Abd al-Rahmán III}

El mismo trabajo permite deducir que este elemento dispone de una planta cuadrada de $5+4 \sqrt{ }_{2}$ varas del Módulo 1 . A partir de ella se desarrollan las distintas alturas en base a un multimódulo de valor $2+\sqrt{ }_{2}$ varas, de manera que la altura del alminar conservado es 8 , la supuesta del primer cuerpo es 11, y la del segundo cuerpo alcanza 14 de estos multimódulos. Sobre este segundo cuerpo, de lado $3+2 \sqrt{ }_{2}$ varas, la media naranja añade otro multimódulo y el remate con esferas se eleva otros 2.

Tanto el ancho de muros como el ámbito de escaleras son de $1+V_{2}$ codos, excepto el machón central que tiene un ancho de 2 codos en la parte conservada y 1 codo en la superior.

La altura de las sillerías es de $2+\sqrt{ }_{2}$ cuartas. Este valor encaja en los tramos dibujados por Félix Hernández, y coincide con la altura que presentan los sillares visibles en la Puerta del Perdón, siendo el ancho del situado junto a la cruz labrada de 1 codo.

Los escalones disponen de una huella de $5 \sqrt{ }_{2}$ palmos y una altura de $2+\sqrt{ }_{2}$ palmos. El fondo de los huecos ciegos de fachada, y el ancho de los rebajes en los testeros de los muros sobre los que se desarrollan ambas escaleras, es de $\sqrt{ }_{2}$ codos. En los laterales de estos muros los 3 rebajes del primer nivel tienen un ancho de 3 codos, y los 4 del resto de plantas es de 2 codos.

\section{MEDICIONES DIRECTAS}

Los patrones construidos con las distintas métricas detectadas han permitido comprobar resultados in situ, así como el análisis directo de elementos no representados en las bases de referencia.

\subsection{Módulo 1 (Figura 5)}

En el interior del templo las basas de las columnas disponen de $\sqrt{ }_{2}$ codos de lado. Además se aprecia que el espesor total de las trazas del antiguo muro de la qibla, perteneciente a la mezquita fundacional de Abd al-Rahmán I y señaladas por Félix Hernández Giménez en la actual solería, es $\sqrt{ }_{2}$ varas.

Los capiteles del mihrab se adaptan a una altura de $\sqrt{2}_{2}$ cuartas. Lo mismo están separados los ejes de las parejas de columnas. La profundidad de sus basas es de 1 cuarta. El fondo del cimacio es $\sqrt{ }_{2}$ codos, y su altura es $2 \sqrt{ }_{2}$ palmos.

En la fachada sur del patio los contrafuertes de la Puerta de las Palmas disponen de una altura regular de sillares de $V_{2}$ cuarta. En otro contrafuerte lateral aparecen trabas con sillares de 1 codo de altura.

\subsection{Módulo 3 (Figura 6)}

En el Postigo de la Leche la profundidad de las jambas es de 1 cuarta y el umbral avanza hasta $\sqrt{ }_{2}$ cuarta del Módulo 3 . Se chequea y comprueba que este mismo módulo fue el utilizado en la construcción del Hospital de San Sebastián, tanto en la sillería de sus muros exteriores como en los elementos de su portada.

La misma métrica parece presentar también la sillería del alzado norte del patio, incluida la Puerta del Perdón y la torre del campanario cristiano. Los sillares presentan dimensiones en codos, cuartas y pies enteros.

\subsection{Módulo 4}

En el interior de la Capilla de la Inmaculada Concepción se detectan varios elementos dimensionados en escala estática base del Módulo 4.

Sin embargo en el Capilla Mayor predominan las modulaciones $\sqrt{ }_{2}$. La altura y separación de las columnillas del sagrario es $\sqrt{ }_{2}$ codos, y sus basas tienen $\sqrt{ }_{2}$ palmos de ancho (Figura 6). La solería en damero es una cuadrícula de $\sqrt{ }_{2}$ cuartas. En otros detalles la modulación responde tanto a palmos enteros como a $\sqrt{ }_{2}$ palmos.

\section{CONCLUSIONES}

Tanto en la mezquita fundacional como en cada una de las ampliaciones realizadas por los distintos gobernantes musulmanes se mantuvo el mismo patrón dimensional correspondiente al Módulo 1. Este bajo valor no se corresponde con ninguno de los codos hispano musulmanes publicados hasta ahora ${ }^{5}$, ni con el de Madinat al-Zahra de 169,92 cm obtenido aplicando este método. Su invariabilidad, a lo largo del dilatado periodo de 2 siglos de evolución de la mezquita aljama, denota el afán de sus constructores por considerarlo un proyecto unitario al mantener la proporcionalidad de las partes añadidas.

En contraste con esta uniformidad métrica destaca la variabilidad del resto de patrones utilizados en las posteriores reformas. Los Módulos 3 y 4, correspondientes sin duda a obras cristianas, tampoco se ajustan a los valores de las varas oficiales conocidas (la antigua vara de Toledo, y la de Burgos o Castellana).

Cierta relevancia histórica puede tener también el resultado obtenido en la Puerta de los Deanes. Por un lado se puede confirmar el origen emiral de sus sillerías interiores. Por otra parte su exterior está realizado con el Módulo 2, que no coincide con el resto de la mezquita. El alto valor del patrón utilizado tampoco se ajusta a los oficiales publicados. El hecho de que esta métrica sólo coincida con el escudo heráldico del obispo Don Juan Daza, que se localiza en el Postigo del Obispo, podría relacionar la reforma exterior de esta puerta con el mandato del obispo, entre 1505 y 1510 . No obstante ha quedado incompleto el estudio de otras partes del edificio que podrían arrojar el mismo módulo.

\footnotetext{
5 Félix Hernández (23) compara las dimensiones de la mezquita ofrecidas por distintas fuentes escritas árabes. Estima que en su construcción se utilizaron dos tipos de medidas, el codo ma'muni «con exacta equivalencia» de 47,14 cm, y el codo rassasi de 58,93 cm. Por otra parte se aportan numerosas denominaciones y valores distintos para el codo hispano-musulmán en (37).
} 


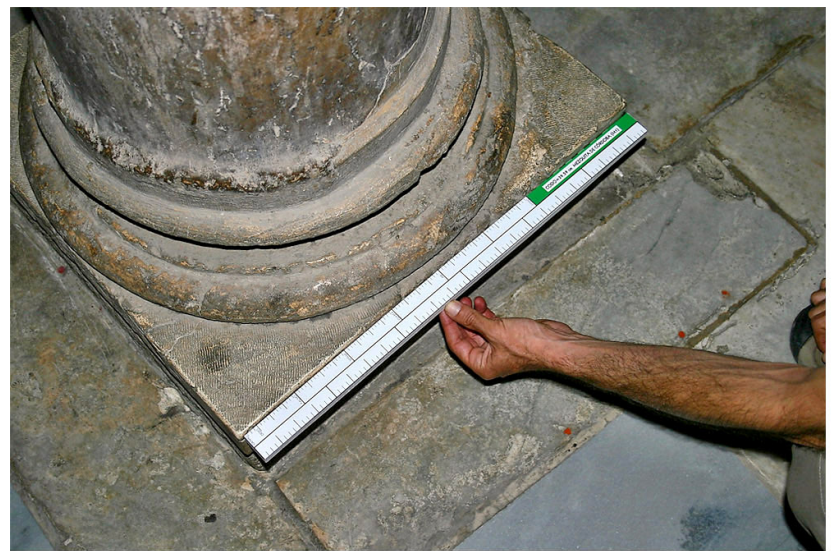

Basa de columna de las arquerías de la mezquita

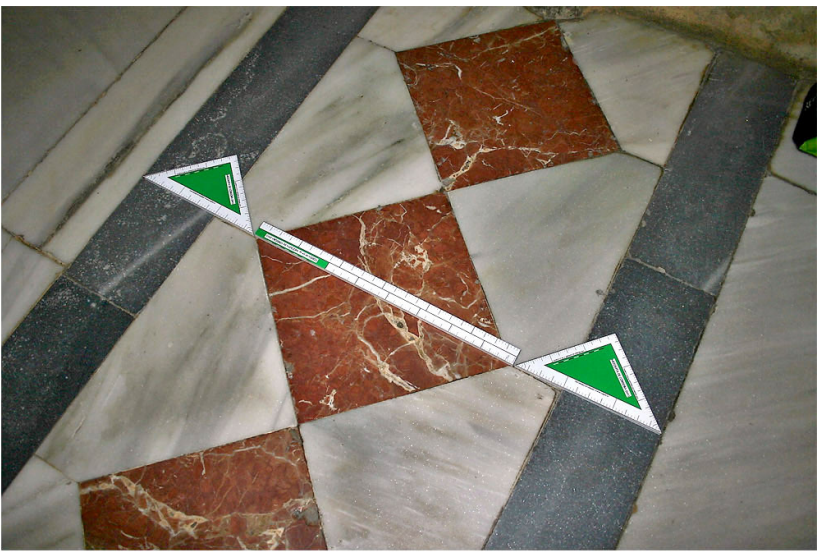

Trazas de la qibla de la mezquita fundacional

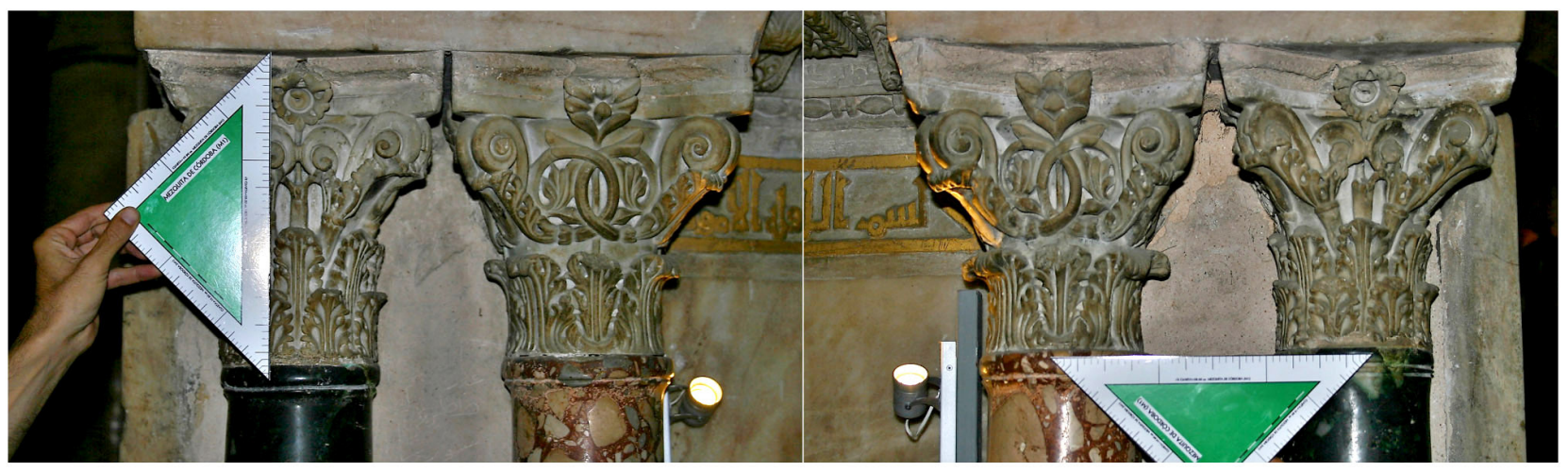

Capiteles del mihrab
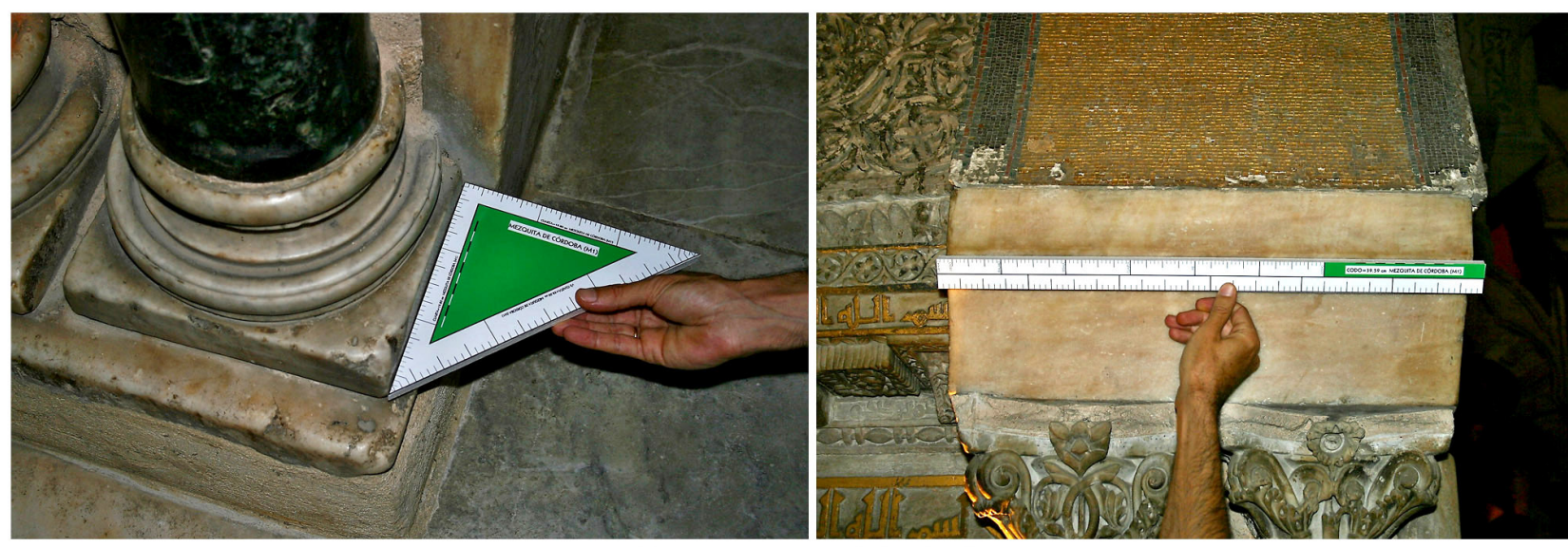

Basa y cimacio del mihrab

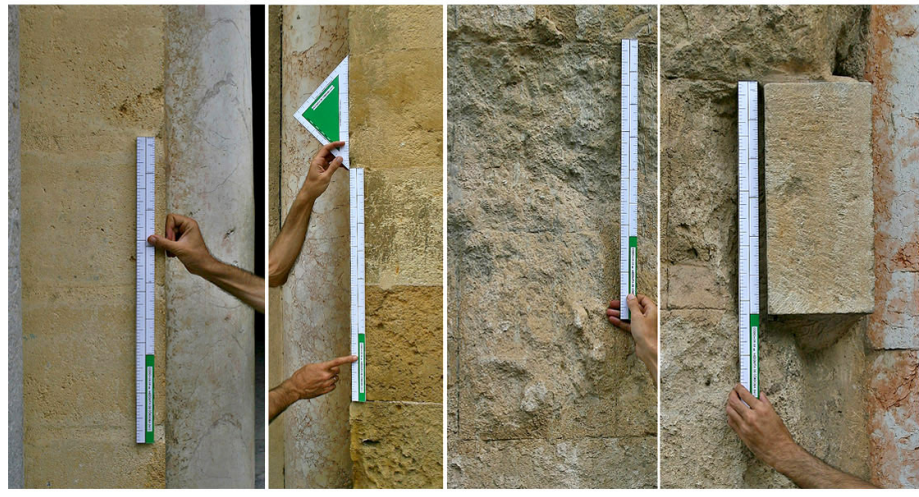

Sillares de la fachada del oratorio al patio



Sillares de la fachada este de al-Mansur

Figura 5. Mediciones directas del módulo 1. 




Hospital de San Sebastian

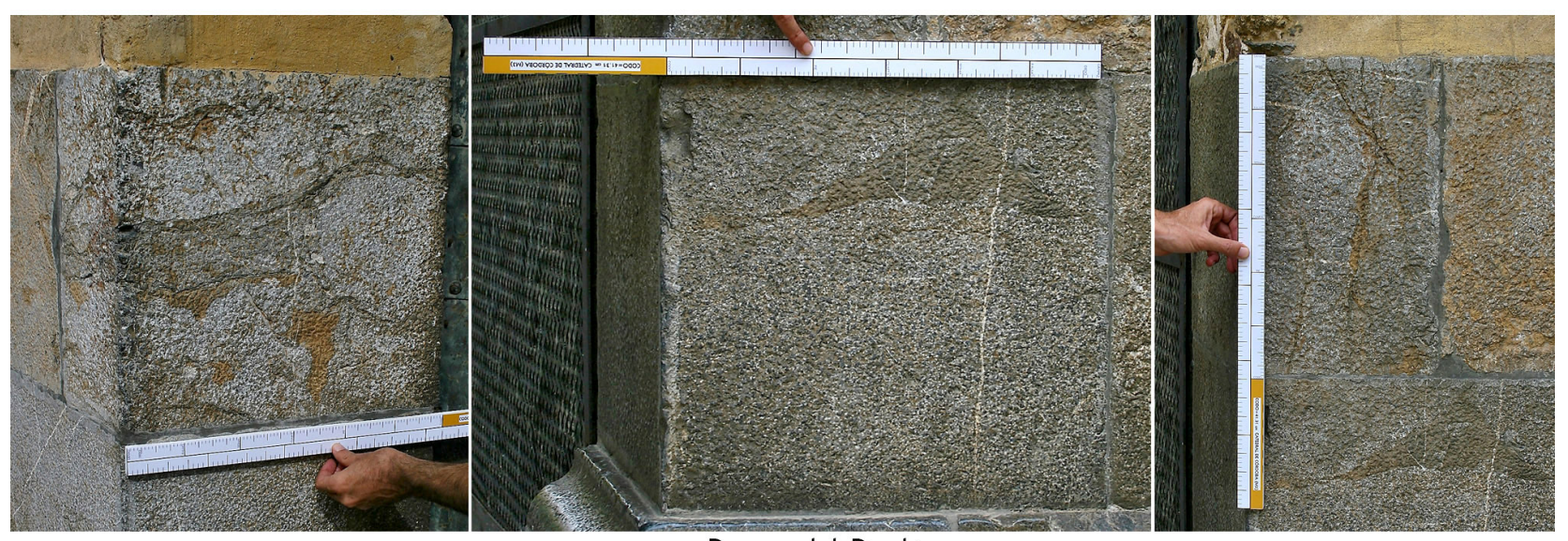

Puerta del Perdón
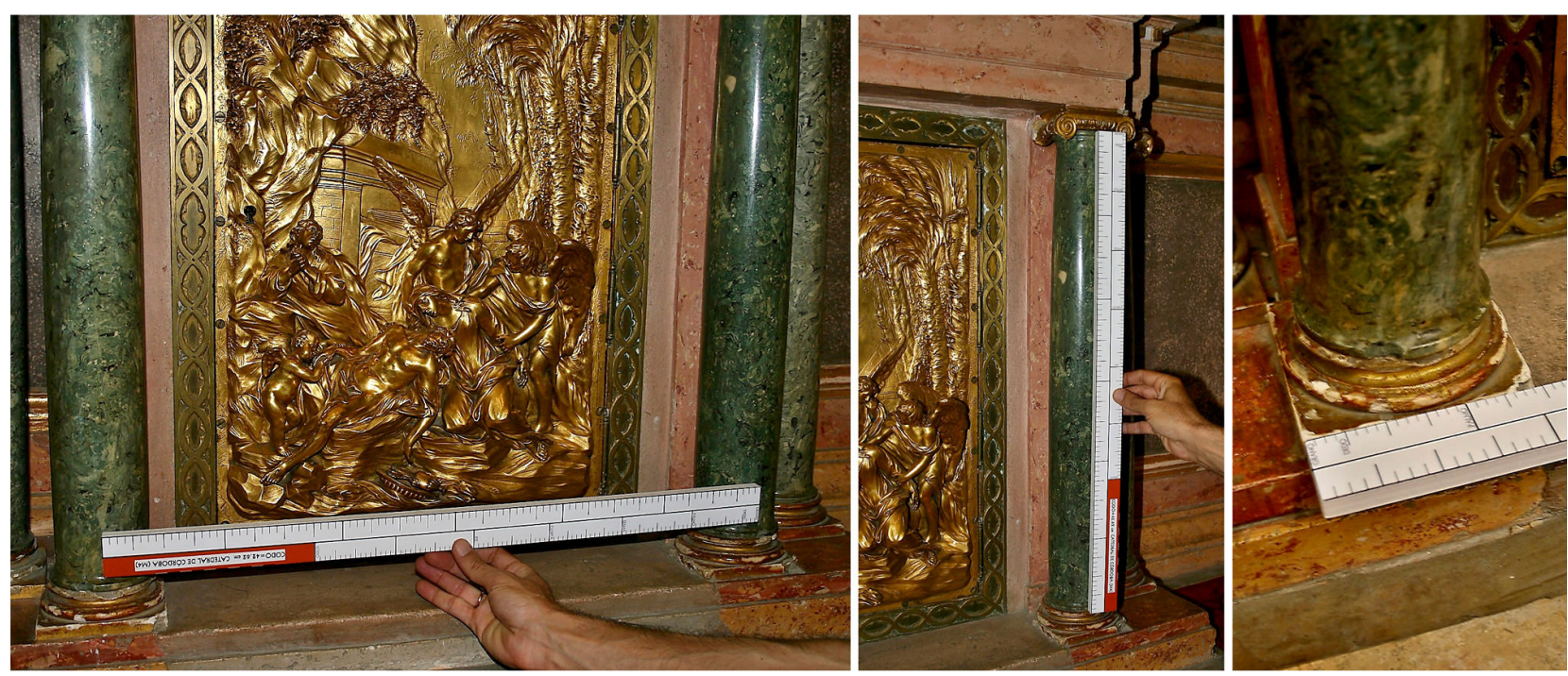

Sagrario de la Capilla Mayor

Figura 6. Mediciones directas de los módulos 3 y 4. 
El Módulo 3 utilizado en el Postigo de la Leche, en el cerramiento exterior del patio, en la Puerta del Perdón, en la torre del campanario y en otras zonas puntuales del alzado oeste, corresponde también con la métrica del Hospital de San Sebastián, obra atribuida a Hernán Ruiz I que utiliza la misma piedra de cantera (36).

Según las crónicas este mismo maestro inició poco después las obras del crucero y naves de la catedral. El hecho de que utilizase el distinto Módulo 4 puede estar relacionado con la necesidad de adaptarse a las dimensiones de la mezquita donde se insertaba. En vez de utilizar el mismo Módulo 1 con que se había construido el templo original, por desconocimiento o intencionadamente, se adopta otro que permite una curiosa compatibilidad geométrica. Como referencia modular debió utilizarse aproximadamente la distancia entre ejes de las naves intermedias. De esta manera las 4 brazas de las naves del coro de la catedral coinciden con los ejes de las naves intermedias de la mezquita. Las $2+3 \sqrt{ }{ }_{2}$ brazas del interior del crucero en sentido este-oeste se adaptan al ancho de dos naves de la mezquita, una intermedia y otra extrema. En sentido norte-sur el crucero dispone de $4+3 \sqrt{ }_{2}$ brazas del Módulo 4, lo que permite que entre los soportes de la nave central, de otra braza de espesor, se abarque el espacio ocupado por 5 arcadas de la mezquita (10 brazas del Módulo 1). El ancho de las naves laterales del crucero de la catedral, dimensionadas mediante $4+V_{2}$ brazas, se ajustan a su vez a 3 de las arcadas de la mezquita.

Por su parte las posteriores obras de la Capilla de la Inmaculada Concepción, la Sala Capitular y la Puerta de Santa Catalina, mantienen el mismo Módulo 4 que el crucero de la Catedral, denotando también aquí la intención de considerar todas estas partes aisladas como un proyecto unitario.

Destaca la distinta distribución del uso de la escala $\sqrt{ }_{2}$ en la planta de los dos templos superpuestos. En la mezquita se enfatiza el eje de la nave central y el espesor de todas las arquerías, mientras que en sentido perpendicular la escala mayor se reserva a la definición del doble muro de oración o qibla produciéndose la intersección en forma de $\mathrm{T}$ en el nicho del mihrab. En cambio en la catedral se sigue el esquema de utilizar la $\sqrt{ }$ centrada en el crucero, formalizándose una cruz latina. También se acude a esta escala en la definición del ancho de las naves laterales.

Mientras que el espesor de los soportes y la altura de las sillerías del templo cristiano se ajustan a la escala base, en la obra musulmana casi todos los muros presentan unas dimensiones determinadas por la utilización única o combinada de la escala $\sqrt{2}_{2}$.

Sorprende observar que mientras en la zona de época emiral, correspondiente a la mezquita fundacional, al alminar de Hisam I, y a la ampliación de Abd al-Rahmán II, la altura de los sillares se ajustan en general a $V_{2}$ codos, en el alminar de Abd al-Rahmán III y en la ampliación de al-Hakam II, de época califal, se adopta una dimensión dinámica de valor $2+V_{2}$ cuartas.

Este criterio adoptado por los alarifes cordobeses no se mantiene en el trazado de las portadas existentes en el alzado oeste. En todos los casos, y a partir al menos del dintel de las puertas, las dimensiones generales de los distintos elementos (incluida la fábrica que los sustenta) se determina sólo me- diante unidades de la escala base. Ello provoca la no concordancia en altura entre las hiladas de sillería perteneciente a los lienzos de muro y las que configuran las distintas portadas. Este hecho queda claramente patente sobre el Postigo del Obispo, en donde debió existir un cuerpo superior original. También se observa en la Puerta de los Visires, si bien en este caso la sillería superior en escala base se extiende hasta los contrafuertes en donde vuelve a adoptar la escala $V_{2}$.

No parece ser este este el caso de la fachada este, correspondiente a la última ampliación de al-Mansur. Según las mediciones directas realizadas, tanto puertas como lienzos comparten una sillería común de modulación estática $\sqrt{ }_{2}$.

\subsection{Proporciones notables}

La Puerta de Pasadizo se localiza entre los muros de la qibla, en un lienzo de sillares de altura $2+\sqrt{ }_{2}$ cuartas. En su umbral se localizan 5 sillares colocados a tizón de 1 codo de ancho. El ancho del hueco es de 1 braza y la altura se ajusta a $5+\sqrt{ }_{2}$ codos, por lo que la proporción obtenida representa una aproximación dinámica al rectángulo áureo.

En las 3 puertas de al-Hakam II el hueco central tiene un ancho de 26 y una altura de $24+15 V_{2}$ palmos (razón alto/ ancho $=1,732)$, que resulta ser una aproximación operativa al rectángulo $\sqrt{ } 3$ (razón 1,739, error del 0,4 \%).

En el resto de huecos centrales no se detectan proporciones singulares. Sin embargo sí es posible encontrar combinaciones de elementos de cada portada que presenten aproximaciones a alguna constante matemática. Por ejemplo, y de nuevo en las puertas de al-Hakam II, las dimensiones del hueco se combinan con las de las jambas y el dintel. En la Puerta de San Ildefonso los 4 palmos de las jambas amplían el hueco a 34 palmos de ancho, que por la altura del hueco produce un marco de proporción 1,330, lo que representa una aproximación a 4/3 con un error del -0,26 \%. Si a esta altura de $24+15 \sqrt{ }_{2}$ palmos le añadimos los $6+3 \sqrt{ }_{2}$ del dintel obtenemos $30+18 \sqrt{2}_{2}$, que entre los 34 de ancho arrojan un valor de 1,631. Es otra aproximación operativa a la divina proporción, dado que el número de oro es aproximadamente 1,618 (error del $0,8 \%)$

Ensayando sobre la trama modular de esta portada se encuentran configuraciones simétricas que presentan buenas aproximaciones a la Proporción Cordobesa, a 4/3, a $\sqrt{ }_{2}$, a la Divina Proporción y a $\sqrt{ } 3$ (Figura 7).

Y si bien estas coincidencias pudieran tener carácter aleatorio y no responder a la intención del proyectista, se resalta el dimensionamiento de distintos elementos de la mezquita de Córdoba en base al número primo 13. Así el ancho de la nave central es de $13 \sqrt{ }_{2}$ codos. El ancho de los lienzos de muro del alzado oeste con portadas es de 26 codos (excepto en la Puerta de los 3 Nombres). El ancho de las 3 puertas de al-Hakam II, y la altura de las ventanas laterales de la Puerta del Espíritu Santo, es 26 palmos.

\section{REFLEXIONES}

Cada obra analizada confirma la hipótesis de que en este procedimiento se basó durante milenios el diseño y la construcción de todo tipo de obras, y permite constatar que el sistema modular detectado es altamente eficiente. 




P. Cordobesa (1.306...)

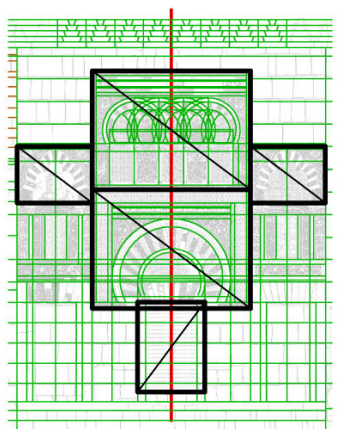

$4 / 3$

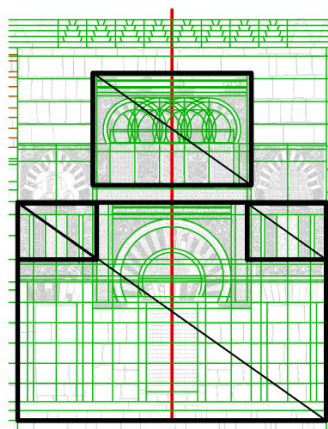

Raíz de 2

$(1.414 \ldots)$

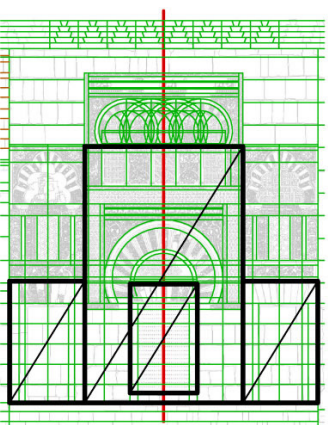

Divina Proporción $(1.618 \ldots)$

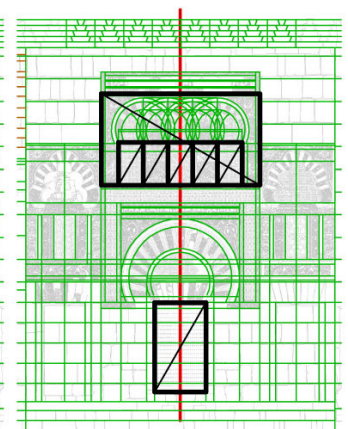

Raíz de 3

$(1.732 \ldots)$

Figura 7. Proporciones notables en la Puerta de San Ildefonso.

Esto ayuda a responder sencillamente a muchas de las cuestiones que sobre la proporción de la arquitectura se han planteado hasta ahora.

También plantea nuevos interrogantes, como por ejemplo:

- ¿Cómo no se ha tenido conocimiento hasta ahora de este sistema modular tan sencillo, práctico, y aparentemente universal?

- ¿Cuándo comenzó a utilizarse? ¿En dónde se usó? ¿Persiste en la actualidad?

- ¿Por qué, si se han planteado tantas hipótesis y teorías, no se había considerado el sistema modular constatado en esta investigación?
- ¿Qué ocurre con los materiales de acarreo que solían reutilizarse en las construcciones?

- ¿Corresponde el Módulo 1 al codo emiral, y el detectado en Medinat al-Zahra al califal?

Afortunadamente el conocimiento del mecanismo de diseño y modulación del pasado, recuperado en apariencia de un secreto ancestral, está permitiendo responder a muchas de estas cuestiones. Conforme avanzan las investigaciones se obtienen nuevos resultados que retroalimentan el conocimiento sobre el propio sistema, y que han sido o serán objeto de nuevas divulgaciones.

\section{REFERENCIAS}

(1) Moya-Blanco, L. (1993). La arquitectura cortés y otros escritos. Madrid: Colegio Oficial de Arquitectos.

(2) Pacioli, L. (1991). La divina proporción. Madrid: Akal.

(3) Neufert, E. (1965). Industrialización de las construcciones: Manual de la construcción racional con medidas normalizadas. Barcelona: Gustavo Gili.

(4) Aguirre-de Yraola, F. (1969). Coordinación dimensional de unidades de obra y elementos de construcción. Madrid: Consejo Superior de Investigaciones Científicas, Patronato de Investigación Científica y Técnica Juan de la Cierva.

(5) Kurent, T. (1985). La coordinación modular de las dimensiones arquitectónicas. Boletín del Museo Arqueológico Nacional, 3(1): 69-95.

(6) Schmitt, H., Heene, A. (1998). Tratado de construcción. Barcelona: Gustavo Gili.

(7) Kula, W. (1980). Las medidas y los hombres. Madrid: Siglo XXI.

(8) Michell,J. (1981). Ancient Metrology. Bristol: Pentacle Books.

(9) Escalona-Molina, M. (2009). Estadal: Una aproximación al universo de la mensura. Sevilla: Junta de Andalucía, Servicio de Publicaciones y Divulgación

(10) Suárez-Jiménez, C. M. (2009). Métrica en arquitectura. México: Universidad Iberoamericana.

(11) Vitrubio-Polión, M., Rodríguez-Ruiz, D., Ortiz y Sanz, J. (2007). Los diez libros de arquitectura. Madrid: Akal.

(12) Evers, B., Thoenes, C. (2011). Teoría de la arquitectura: Del renacimiento a la actualidad. Koln: Taschen.

(13) Wittkower, R. (1968). La arquitectura en la edad del Humanismo. Buenos Aires: Nueva Visión.

(14) Moya-Blanco, L. (1981). Relación de diversas hipótesis sobre las proporciones del Partenón. Academia: Boletín de la Real Academia de Bellas Artes de San Fernando, 52: 25-156.

(15) Ruiz de la Rosa, J. A. (1987). Traza y simetría de la arquitectura: en la Antigüedad y Medievo. Sevilla: Universidad de Sevilla.

(16) Fernández-Gómez, M. (1999). La teoría clásica de la arquitectura: clasicismo y renacimiento. Valencia: Universidad Politécnica.

(17) Roldán-Medina, F.J. (2014). Principios de metrología en la arquitectura del pasado. Granada: Universidad de Granada.

(18) Orti-Belmonte, V. (1929). Guía artística de Córdoba. Córdoba: Rogelio Luque.

(19) Nieto-Cumplido, M. (1998). La catedral de Córdoba. Córdoba: Cajasur.

(20) Ruiz-Cabrero, G. (2009). Dibujos de la Catedral de Córdoba: visiones de la Mezquita. Córdoba: Cabildo Catedral.

(21) Marfil-Ruiz, P. (2010). Las puertas de la Mezquita de Córdoba durante el Emirato Omeya. Córdoba: Universidad de Córdoba. 
(22) Camps-Cazorla, E. (1953). Módulo, proporción y composición en la arquitectura califal cordobesa. Madrid: Instituto Diego Velázquez.

(23) Hernández-Giménez, F. (1961). El codo en la historiografía árabe de la Mezquita Mayor de Córdoba: contribución al estudio del monumento. Madrid: Maestre.

(24) Hoz-Arderius, R. (2002). La proporción cordobesa. Córdoba: Colegio de Arquitectos.

(25) Merino de Cáceres, J.M. (1985). El trazado de la Bab Al-Uzara (Puerta de San Esteban en la Mezquita de Córdoba). Academia: Boletín de la Real Academia de Bellas Artes de San Fernando, 60:287-298.

(26) Fernández-Puertas, A. (2000). Mezquita de Córdoba. Trazado proporcional de su planta general (siglos VIII-X). Archivo Español de Arte, 73(291):217-247, doi: http://dx.doi.org/10.3989/aearte.2000.v73.i291.829.

(27) Fernández-Puertas, A. (2008a). Mezquita de Córdoba: 'Abd al-Raḥmān I (169/785-786). El trazado proporcional de la planta y alzado de las arquerías del oratorio. La qibla y el mihrāb del siglo VIII. Archivo Español de Arte, 81(324): 333356, doi: http://dx.doi.org/10.3989/aearte.2008.v81.i324.127.

(28) Fernández-Puertas, A. (2008b). Otro trazado de la Bab al-Uzara. Miscelánea de estudios árabes y hebraicos. Sección Arabe-Islám, 57: 75-121.

(29) Fernández-Puertas, A. (2009). Mezquita de Córdoba: el trazado de la portada interior de la Bāb al-Wuzarā'. La Puerta de los Deanes (s. VIII), su trazado interior y exterior. Archivo Español de Arte, 82(326): 107-136, doi: http://dx.doi. org/10.3989/aearte.2009.v82.i326.146.

(30) Klein, H.A. (1974). The world of measurements: masterpieces, mysteries and muddles of metrology. Nueva York: Simon and Schuster.

(31) Almagro-Gorbea, A. (1985). La fotogrametría, un valioso auxiliar para el estudio de la arquitectura islámica. Cálamo. Revista de Cultura Hispano-Árabe, 6: 14-18.

(32) Almagro-Gorbea, A. et al. (1996). Arquitectura en al-Andalus: Documentos para el siglo XXI (p. 207). Barcelona: Lunwerg.

(33) Roldán, F. (2011). La escuadra sagrada. Madrid: Bubok Publishing S.L.

(34) Roldán, F. (2012a). Modulación, tipología de tramas y ajuste a los límites en la metrología arquitectónica clásica. EGA: Revista de Expresión Gráfica Arquitectónica, (20): 172-183, doi: http://dx.doi.org/10.4995/ega.2012.1448.

(35) Roldán, F. (2012b). Method of modulation and sizing of historic architecture. Nexus Network Journal, 14(3): 539-553. doi: http://dx.doi.org/10.1007/s00004-012-0125-5.

(36) Marfil-Ruiz, P. (2010). Las puertas de la Mezquita de Córdoba durante el Emirato Omeya (p. 577). Córdoba: Universidad de Córdoba.

(37) Vallvé-Bermejo, J. (1976). Notas de metrología hispano-árabe. El codo en la España musulmana. Al-Andalus: revista de las Escuelas de Estudios Árabes de Madrid y Granada, 41(2): 339-354. 